\title{
Gewerkschaftliche Kämpfe gegen Betriebsschließungen - Ein Anachronismus?
}

\author{
Richard Detje, Wolfgang Menz, Sarah Nies \\ Günter Sanné, Dieter Sauer
}

\begin{abstract}
Wenige Monate nachdem mit der AEG in Nürnberg ein Symbol deutscher Industriegeschichte verschwand, wird in Bochum bei Nokia die Perspektive eines schöpferischen Strukturwandels demontiert. Beides sind herausragende Beispiele für eine wachsende Zahl von Betriebsschließungen, bei denen mit den Arbeitsplätzen auch soziale Lebensverhältnisse zerstört werden. Doch Belegschaften, Betriebsräte und Gewerkschaften organisieren Widerstand. Es entwickeln sich Kämpfe gegen Betriebsschließungen und Standortverlagerungen. Der Erfolg solcher Gegenwehr erschließt sich nicht allein über ihre materiellen Ergebnisse und den Erhalt von Arbeitsplätzen. Es geht vielmehr auch um Ansprüche an Beteiligung und um den Anstoß von weiterreichenden Politisierungs- und Mobilisierungsprozessen.
\end{abstract}

\section{Einleitung}

Betriebsschließungen und Standortverlagerungen gehören zum kapitalistischen Alltag. Rund 27.500 Betriebe meldeten im vergangenen Jahr Insolvenz an. 440.000 Arbeiter und Angestellte verloren dadurch ihren Arbeitsplatz. Dennoch gehen Insolvenzen in der weit überwiegenden Zahl geräuschlos über die Bühne. Zum einen, weil das Gros der Fälle Kleinst- und Kleinbetriebe sind. Zum anderen, weil die Situation ausweglos zu sein scheint: Wegbrechen des Absatzes, Verluste, Aufzehrung des Eigenkapitals, Überschuldung. Doch über diesen basso continuo haben sich in den letzten Jahren Melodien gelegt, die Neues verkünden.

Die eine Melodie spielen das Management und die Eigentümer. BetriebsschlieBungen erfolgen heute in einem ökonomischen Umfeld, das durch radikalisierte Renditeansprüche geprägt wird. Um diese zu befriedigen, werden massive Restrukturierungsprogramme durchgeführt, zu denen auch Betriebsschließungen und Standortverlagerungen gehören. Die andere Melodie spielen die Beschäftigten und ihre Interessenvertretungen, die betriebswirtschaftlich begründete Arbeitsplatzvernichtung nicht fatalistisch als Marktzwang hinnehmen, sondern Widerstand organisieren.

Über diesen Widerstand gibt es bisher nur punktuelle Berichte und wenige, in der Regel den Einzelfall beleuchtende Dokumentationen. Doch was dokumentiert das Besondere, was das möglicherweise Para- digmatische? Wie ist es in diesem ,worst case" von Interessenvertretung um deren Handlungsmöglichkeiten bestellt?

Ulrich Beck hält gewerkschaftliche Kämpfe gegen Betriebsschließungen schlicht für einen Anachronismus, wenn nicht gar für eine Verzweiflungstat: „Bei den Gewerkschaften haben wir es mit entkernten Institutionen $\mathrm{zu}$ tun, die Fassade ist geputzt, dahinter steht nichts mehr. Die haben keine Ahnung, wie sie die neuen groBen Herausforderungen meistern sollen. Man kann das zum Beispiel bei den Protesten gegen Werkschließungen bei Opel oder Infineon sehen. Gegen diese Schließungen haben Gewerkschaften Streiks organisiert, was mir unendlich absurd vorkommt: Die Arbeiter, deren Arbeit nicht mehr gebraucht wird, verweigern sich zu arbeiten" (Beck 2007, S. 240).

Für Mario Candeias und Bernd Röttger ereignet sich im Widerstand gegen Betriebsschließungen dagegen so etwas wie eine , kleine Revolution“. Denn jenseits der ausgetretenen Pfade des dualen Systems der Interessenvertretung entstehe „eine neue >Kultur der Beteiligung “" (Candeias/ Röttger 2007, S. 94), mit der Ohnmachtserfahrungen abgeschüttelt würden und kollektives Selbstbewusstsein entstehe.

Anachronismus oder Zeitenwende? Um das beurteilen zu können, brauchen wir über Einzelfälle hinaus aufbereitete Empirie. Da diese nicht vorliegt, haben wir eine erste Dokumentation aktueller Fälle von Betriebsschließungen in der Metallund Elektroindustrie erstellt, auf die sich die nachfolgenden Befunde und Thesen stützen. ${ }^{1}$

\section{Betriebsschließungen und neue Unternehmens- strategien}

Welchen Charakter und welche Folgen Betriebsschließungen und Standortverlagerungen haben, hängt vom sozioökonomischen Kontext ab, in dem sie stattfinden. Wir unterscheiden im Folgenden drei Konstellationen.
Richard Detje und Günter Sanné sind Mitglieder der Wissenschaftlichen Vereinigung für Kapitalismusanalyse und Gesellschaftspolitik (Wissentransfer), die als Arbeitszusammenhang von Gewerkschaftern und Wissenschaftlern Workshops, Tagungen, Publikationen, Expertisen durchführt. Wolfgang Menz, Sarah Nies und Prof. Dr. Dieter Sauer sind Sozialforscher am Institut für Sozia/wissenschaftliche Forschung e. V., (ISF) München. Arbeitsschwerpunkte: Reorganisation von Unternehmen, Subjektivierung und Vermarktlichung von Arbeit, Leistungs- und Gesundheitspolitik. e-mail: dieter.sauer@isf-muenchen.de 


\subsection{STRUKTURWANDEL UND KRISE}

In den 1960er und frühen 1970er Jahren fanden Betriebsschließungen - Beispiel Zechenstilllegungen - im Rahmen eines dynamischen ökonomischen und gesellschaftlichen Strukturwandels statt. In einem Prozess innerer Landnahme wurde der traditionelle Sektor von der expandierenden industriellen Produktion und den privaten wie öffentlichen Dienstleistungssektoren weitgehend absorbiert (Lutz 1984). Negative Arbeitsmarktfolgen konnten durch unternehmensinterne Versetzungen oder durch Mobilität in wachsende Arbeitsmärkte weitgehend verhindert werden.

Mit der Krise 1974/75 war diese Entwicklungsetappe beschleunigter Kapitalakkumulation vorbei. In einem krisenhaften Strukturwandel „verursachen primär Branchenkrisen, Deindustrialisierungsprozesse und generell eine schwache Nachfrage den Niedergang von Betrieben“ (Heseler 1990, S. 411). Nicht zuletzt, weil sich die Arbeitsmarktbedingungen rapide verschlechterten, wurde die Werften- und Stahlkrise von massiven öffentlichen Protesten, Demonstrationen, Streiks bis hin zu Betriebsbesetzungen begleitet.

In den 1990er Jahren war der krisenhafte Strukturwandel überlagert von den Folgen der deutschen Einheit. Sie leitete einen Deindustrialisierungsprozess ein, der nicht mehr nur einzelne Betriebe oder Branchen betraf, sondern die gesamte Wirtschaft der einstigen DDR. Betriebsschließungen waren so in einen gleichsam nationalen Kontext eingebunden.

Solche Entwicklungsverläufe gab es nicht nur in der Vergangenheit, sie finden auch in der Gegenwart statt, und nicht nur auf der Unternehmensebene, sondern auch in branchenweiten Dimensionen. Wenn auch nicht direkt mit der Kohle-, Stahlund Werftenkrise vergleichbar, so zeigt doch der Niedergang der Handy-Produktion in Deutschland, wie ein Prozess „schöpferischer Zerstörung “ in kurzer Zeit in die Alltäglichkeit der Krise münden kann: Bereits in den 1990er Jahren war Hagenuk, im Jahr 2000 Bosch aus dem Handy-Geschäft ausgestiegen. Spektakulär folgten 2006 die Schließung der erst zwei Jahre zuvor von Siemens übernommenen Handy-Produktion durch BenQ und 2007 das „Aus“ für das „ehemals modernste Handy-Werk Europas" in Flensburg durch Motorola. Und stets traf es auch Zuliefer- betriebe: Yageo in Elmshorn, einen Betrieb, der Siemens, Nokia und Ericsson mit elektronischen Bauteilen beliefert hatte und 2003 geschlossen wurde, oder Quips, dessen Service 2005 auf den Standort Bamberg konzentriert wurde.

\subsection{SCHLIEßUNGEN TROTZ SCHWARZER ZAHLEN}

Auch heute sind viele Betriebsschließungen krisenbedingt. Neu ist, dass ein großer Teil der von uns dokumentierten Betriebe zum Zeitpunkt der Konzernentscheidung zur Stilllegung schwarze Zahlen geschrieben hat. Es handelt sich weder um Insolvenzen bzw. Sanierungsfälle noch um Opfer einer umfassenderen Marktbereinigung. Die nachfolgenden Fälle kennzeichnet, dass die Schließung rentabler Betriebe $\mathrm{zu}$ einem Instrument unternehmenspolitischer Restrukturierung geworden ist.

(1) Opfer einer „Konzentration auf das Kerngeschäft" wurde die CNH-Baumaschinen GmbH in Berlin-Spandau, die unter dem Firmennamen Orenstein \& Koppel auf eine 130-jährige industrielle Tradition zurückblicken konnte. Mehrheitsaktionär wurde 1998 die zu FIAT gehörende New Holland, der weltweit drittgrößte Hersteller von Bau- und Landmaschinen. Mit der Krise bei FIAT begann der Abstieg: Die gut verdienende Konzerntochter $\mathrm{CNH}$ wurde zur cash cow für das angeschlagene automobile Kerngeschäft. Gewinne wurden abgeführt, Investitionen zurückgefahren, eine eigenständige Marken- und Vertriebspolitik aufgegeben. Auch ein 107 Tage währender Streik - der längste in der Berliner Nachkriegsgeschichte - konnte die FIATSpitze von der Betriebsschließung 2006 nicht abbringen. Ein rentables Werk fiel der Restrukturierung eines transnationalen Unternehmens zum Opfer.

(2) Auf eine Umsatzrendite von $5 \%$ brachte es im Jahr 2003 das Bosch Werk in Leinfelden bei Stuttgart. Das war dem Management nicht genug. Mit dem Unternehmenskonzept „Profit 05“ sollte die Umsatzrendite in zwei Jahren auf 7-8 \% gesteigert werden. Um dies durchzusetzen, wurde die Belegschaft vor die Alternative gestellt: Standortverlagerung oder Einführung der 40-Stunden-Woche und Lohnsenkungen. Eine Drohkulisse, die gerade in Großbetrieben der Metall- und Elektroindustrie häufig aufgebaut wird. Das Ergebnis: Ar- beitszeitflexibilisierung, Abbau von Prämien und übertariflichen Zulagen gegen Beschäftigungssicherung und Investitionen zur Profilierung von Leinfelden als Leitwerk und Kompetenzzentrum: Standortsicherung durch renditeorientiertes cost-cutting.

(3) Elektrolux - seit 1994 Eigentümer der AEG - gehört zur börsennotierten Wallenberg-Holding mit dem bezeichnenden $\mathrm{Na}$ men Investor, die u.a. auch an Saab, Scania, Ericsson, ABB und der SEB-Bank beteiligt ist. „Elektrolux ist ein Konzern, der sich in allererster Linie an den Interessen seiner Aktionäre und an der Wertsteigerung ihres Vermögens orientiert. Das Management ... sieht in der Steigerung des >shareholder value seine zentrale Aufgabe" (Bierbaum 2006, S. 93). Die AEG machte in Nürnberg zum Zeitpunkt des Schließungsbeschlusses Gewinne. Die Kapitalrendite reichte jedoch nicht aus, um die Aktienkurse weiter nach oben zu treiben. Für ein finanzmarktgetriebenes Management ein überzeugender Grund, gegen massiven Widerstand einen Traditionsstandort zu schließen.

(4) Otis Elevator mit Sitz in Farmington (Connecticut, USA) ist der weltweit größte Hersteller von Aufzügen, Fahrtreppen und Fahrsteigen. „Otis Deutschland schreibt stattliche schwarze Zahlen, wies für 2001 eine Umsatzrendite von $14,8 \%$ aus. Doch die Zentrale in den USA will jedes Jahr den Gewinn um einen Prozentpunkt steigern ... „Wir sind ein Unternehmen, das nach dem Prinzip des Shareholder value operiert", sagte Otis-Chef Ari Bousbib ... Und er merkte an: „Ich konzentriere mich darauf, Produktionsstätten in kostenintensiven Regionen zu schließen“ (Otis 2005 S. 13). 2004 war das Werk in Stadthagen bei Hannover an der Reihe - es wurde nach Tschechien verlagert.

(5) Der Handy-Weltmarktführer Nokia, zu $25 \%$ im Besitz institutioneller Investoren, weist im letzten Geschäftsjahr eine Umsatzrendite von $22 \%$ aus, im Bochumer Werk liegt sie nach Schätzung der örtlichen IG Metall bei $15 \%$. Nachdem die Produktion in Bochum zwischen Juli und Dezember 2007 mit Sonderschichten und Überstunden noch kräftig hochgefahren worden war, erfuhr die Belegschaft Mitte Januar 2008 vom Vorstandsbeschluss, den Standort zu schließen. Die langjährige Strategie des cost-cutting mit weitestge- 
hender Arbeitszeitflexibilisierung sah das kapital- und finanzmarktorientierte Management offenkundig als erschöpft an.

\subsection{FINANZMARKTORIENTIERTE UNTERNEHMENSSTEUERUNG}

In unserer Dokumentation von Betriebsschließungen überwiegen jene Fälle, bei denen die in der Produktion erwirtschaftete Rendite - sei es im konzerninternen Benchmarking oder im Branchenvergleich - als unzureichend angesehen wird. Diese Produktionsstätten werden nicht vorrangig nach ihren Innovationspotenzialen und ihrer technischen bzw. arbeitsprozesslichen Exzellenz bewertet. Sie werden zunehmend als Kapitalanlagen betrachtet, die in Konkurrenz zu Geldkapitalgeschäften auf den Finanzmärkten stehen. Desinvestment oder Verlagerung an profitabler erscheinende Standorte werden somit zu Instrumenten finanzmarktorientierter Restrukturierung. Schauen wir uns vier Pfade finanzmarktorientierter Unternehmensrestrukturierung kurz an.

(1) Low road: Wo mit einer innovationsund qualitätsorientierten Produktionsund Marketingstrategie in Abgrenzung zum Massenmarkt eine steigende Rendite erzielt werden kann - ein Beispiel ist Bosch Power Tools - wird durchaus auf einer high road industrieller Entwicklung gefahren. Andererseits tendieren finanzmarktorientierte Unternehmen vielfach zu einer Politik der Markteroberung durch Kostenführerschaft, die ohne Rücksicht auf Kompetenzen und Markennamen auf der low road mündet; die also auf Konfektionierung, Standardisierung und economies of scale setzt (vgl. Iwer 2006, S. 84ff.). Diese Strategie verfolgte Elektrolux bei der AEGSchließung, in Ansätzen auch Otis in Stadthagen (der „Mercedes von Otis“) und Kone in Hattingen. Versuche der Betriebsräte und der Gewerkschaft, eine profilierte Markenstrategie wieder ins Spiel zu bringen, prallten an der „billiger und profitabler"-Strategie ab.

(2) Marktbereinigung: In einem ökonomischen Umfeld, das durch eine strukturelle Überakkumulation gekennzeichnet ist, macht es Sinn, Konkurrenten zu übernehmen oder strategische Allianzen zu schmieden. Ersteres war die Strategie von Lone Star, einer Private-Equity-Gesellschaft, die 2005 die Fahrradproduktion von Bike Sys- tems mit Werken in Neukirch/Sachsen und Nordhausen übernahm, um sie 2006 und 2007 mit dem Ziel der Marktbereinigung zugunsten der Mifa (Mitteldeutsche Fahrradwerke) zu schließen. Auf die zweite Variante, die Bildung strategischer Allianzen, ist Alstom im europäischen Kraftwerksmarkt orientiert.

(3) Verlagerung: Ein Kennzeichen finanzmarktorientierter Unternehmenssteuerung ist die Verflüssigung der raum-zeitlichen Fixierungen von Investitionen im Prozess der Globalisierung (Harvey 2005). „Der neue Globalisierungsschub betrifft nahezu alle industriellen Kernbereiche ... Eine Vorreiterrolle scheint dabei die Elektronikindustrie zu spielen“"(Voskamp 2005, S. 116). Die Nokia-Verlagerung nach Rumänien ist unter zwei Gesichtspunkten aufschlussreich: Erstens, weil sie gegenläufig zur Annahme erfolgt, industrielle Wertschöpfung sei durchgängig als global sourcing zu organisieren. Kennzeichen des „Nokia-Weges“ ist Netzwerkbildung: „Ein wesentlicher Wettbewerbsfaktor in der Handyproduktion ist heute die Logistik und das symbiotische Zusammenspiel mit den Zulieferern. Diese wollten aber aus Kostengründen nicht in ausreichender Zahl nach Bochum kommen" (so der Nokia-Vorstandsvorsitzende Kallasvuo). Zweitens, weil Rumänien nicht einfach ein $\mathrm{x}$-beliebiger Niedriglohnstandort ist, sondern ,skills“ einbringt: qualifizierte Arbeitskräfte und F+E-Kapazitäten.

Es ist ein gängiges Vorurteil, dass Verlagerungen aufgrund betriebswirtschaftlicher Schwäche vorgenommen werden. „82\% der befragten Betriebe, in denen eine Verlagerung des Standorts diskutiert wird, beschreiben ihre wirtschaftliche Situation als gut oder sogar sehr gut. Es geht ihnen vergleichsweise besser als Betrieben, die keine Standortverlagerung diskutieren" (Ahlers et al. 2007, S. 54). Das hat einen nahe liegenden Grund: Verlagerungen erfordern Neuinvestitionen, die wirtschaftlich prekäre Unternehmen mit aufgezehrtem Eigenkapital und problematischer Kreditwürdigkeit nur schwer finanzieren können.

(4) Ausreizen von cost cutting: Wo sich hochgeschraubte Renditeerwartungen langfristig aus industrieller Wertschöpfung nicht generieren lassen, wird die Ankündigung von Betriebsschließungen zur Erpressungsstrategie. Nochmals Nokia, dessen Bochumer Standort schon einmal 2001 zur Dis- position gestellt worden war, und wo der Personalkostenanteil in der Fertigung unter $5 \%$ gedrückt wurde: „Wir haben die Kennzahlen verbessert, die Kosten reduziert. Wir haben Arbeitszeiten geschaffen gegen den Widerstand vieler Kollegen, die dazu beigetragen haben, dass wir noch sonntags dreischichtig arbeiten können, dass wir drei bis sieben Tage arbeiten können und dass es Arbeit auf Zuruf gibt", so die Betriebsratsvorsitzende Gisela Achenbach (Hamburger Abendblatt, 28.1.08). Renditeorientiertes cost-cutting stößt jedoch nach zwei Seiten an Grenzen: Zum einen bei der Belegschaft, wenn diese die Erfahrung machen muss, dass Konzessionen immer weniger mit belastbaren Standortgarantien honoriert werden. Zum andern auf Seiten des Managements, dessen Renditevorgaben immer unrealistischer werden, je mehr die Zitrone Belegschaft bereits ausgepresst ist. Was englische Fabrikinspektoren im 19. Jahrhundert die „kleinen Diebstähle" des Kapitals durch das "nibbling and cribbling at meal times" (Marx 1972, S. 257) genannt haben, findet in der finanzmarktorientierten Unternehmenssteuerung eine moderne Form. An allen Stellen - bei den Löhnen und Arbeitszeiten, den Preisen der Zulieferer, den Steuern und Subventionen - wird geknabbert und geknapst, um die Rendite zu erhöhen.

\section{Aufkündigung der Sozialpartnerschaft}

Betriebsschließungen sind zumeist vorläufige Endpunkte im Restrukturierungsprozess eines Unternehmens. Als solche weisen sie eine Vorgeschichte auf, die eine zunehmende Abkehr von einem konsens- und vertrauensorientierten Arrangement der industriellen Beziehungen anzeigt.

(1) Der Grundsatz, dass Verträge einzuhalten sind, erodiert in den Managementetagen. Betriebliche Vereinbarungen über Beschäftigungs- und Standortsicherung werden vorfristig aufgekündigt oder zur Neuverhandlung mit verschlechterten Konditionen für die Belegschaft aufgerufen. Nicht selten erfolgt die Stilllegungsplanung entgegen vorheriger Vereinbarungen eines Interessenausgleichs und Sozialplans, wie bei ThyssenKrupp Drauz-Nothelfer. 
(2) Informations- und Mitbestimmungsrechte des Betriebsrats werden unterlaufen. So ergibt die Auswertung der WSI-Betriebsrätebefragung, dass in Betrieben mit 100-499 Beschäftigten Standortverlagerungen in erheblichem Umfang ohne vorhergehende Diskussion stattfinden (Ahlers et al. 2007, S. 53). Auch die mangelhafte Qualität der Informationspolitik und die Kurzfristigkeit scheinen in einer wachsenden Zahl von Fällen auf eine Strategie der Überrumpelung der Interessenvertretung angelegt zu sein („Fakten schaffen“).

(3) Organisatorische Dezentralisierung geht mit strategischer Zentralisierung im Unternehmen einher. Wo dies der Fall ist, kann von einer "Mitbestimmungskultur“ vor Ort oft keine Rede sein, da das lokale Management über keine (ausreichenden) Vollmachten und Handlungsspielräume verfügt, wie beispielhaft die Betriebsschließungen von Kone in Hattingen und $\mathrm{CNH}$ in Berlin-Spandau gezeigt haben.

(4) Dass sich Unternehmensleitungen zunächst weigern, überhaupt in Verhandlungen mit Betriebsrat und Gewerkschaft einzutreten, ist keine Seltenheit. Das Pochen auf die Eigentums- und Direktionsrechte hat auch in einem Unternehmen wie Bosch Verhandlungstraditionen zweifelhaft werden lassen. Organisierter Widerstand der Belegschaft wird erforderlich, um Verhandlungen durchzusetzen. Dagegen setzen Unternehmensleitungen immer häufiger auf einen frühen Konfrontationskurs, wie bei Panasonic in Esslingen mit einstweiliger Verfügung gegen Betriebsversammlungen und Sanktionsandrohungen gegen Beschäftigte, die an Protesten und Demonstrationen gegen die Schließung ,ihres“ Betriebs teilnahmen.

Der Typus von Betriebsschließungen, wie er für den finanzmarktorientierten Kapitalismus kennzeichnend ist, stellt die Belegschaften und ihre betriebliche und gewerkschaftliche Interessenvertretung vor neue Handlungsbedingungen. Eingespielte sozialpartnerschaftliche Arrangements werden einseitig aufgekündigt, co-management- und konsensorientierte Interessenvertretungspolitiken laufen zunehmend ins Leere. einer Beschäftigungs- und Qualifizierungsgesellschaft sowie Weiterbeschäftigung bzw. Altersteilzeit von knapp 200 Beschäftigten durchgesetzt (Infineon 2006, S. 18).

Vierter Irrtum: Über den materiellen Erfolg hinaus müssen elementare Grundsätze kollektiven Widerstands erkämpft werden: Durchsetzung des Streikrechts und des Rechts auf eine autonome Interessenvertretung, Verhinderung der Spaltung der Belegschaft wie auch einer Spaltung von betrieblicher und gewerkschaftlicher Interessenvertretung.

Ulrich Beck irrt also auch in grundsätzlicher Hinsicht. Es geht nicht um Fassadenputz, sondern um Grundsatzfragen gewerkschaftlicher Interessenvertretung. Es geht darum, wie weit dem Recht auf Arbeit in einer Produktionsweise, die auf der Dispositionsfreiheit des Kapitaleigentums basiert, Geltung verschafft werden kann. Dort, wo Recht wider Recht steht, entscheidet der soziale Konflikt.

Anhand unserer Falldokumentation lassen sich vier typische Formen von Konfliktverläufen unterscheiden, die durch unterschiedliche Zielsetzungen und Handlungsstrategien der Akteure und durch jeweils spezifische Akzentsetzung in der Wahl der Kampfinstrumente geprägt sind.

\subsection{VERHANDLUNGEN UM ÖKONOMISCHE ALTERNATIVKONZEPTE}

Diese Strategie zielt darauf, gegen die Schließungspläne ein tragfähiges ökonomisches Alternativkonzept zu entwickeln, das mit der Unternehmensseite zu verhandeln ist. Angesichts der verschlechterten industriellen Beziehungen erfordert dieser Weg die glaubwürdige Demonstration von Druckpotenzialen. Neben Arbeitsniederlegungen und Protesten, die auch die Öffentlichkeit mobilisieren sollen, sind dies: ausgedehnte Informationsveranstaltungen des Betriebsrats, über Tage sich erstreckende Betriebsversammlungen, Vernetzung mit Betrieben im regionalen Umfeld bzw. innerhalb der Wertschöpfungsketten. So wurden in der erfolgreichen Auseinandersetzung um die Schließung von Nordgren in Großbettlingen die ökonomisch außerordentlich einflussreichen Kundenbetriebe (u.a. Daimler, Volvo, ZF) einbezogen, die einen Verzicht auf die Schließung und Verlagerung durchsetzten.

Beispiel für eine sanktionsbewehrte Verhandlungsstrategie ist Kaltenbach \& 
Vogt (KaVo) in Leutkirch. Die Schließungsabsicht des Finanzinvestors Danaher im November 2004 traf den Betriebsrat zunächst vollkommen unerwartet, da KaVo seinerzeit florierte. Allerdings lag die Rendite mit $10 \%$ deutlich unter den Erwartungen des Investors, der $18 \%$ anpeilte. Betriebsrat und IG Metall konzentrierten sich von Anfang an auf eine verhandlungsorientierte Strategie, die durch eine breite Mobilisierung, vielfältige Aktionen der Belegschaft und der Öffentlichkeit unterstützt wurde. Und sie erarbeiteten in mehreren Verhandlungsschritten ein Alternativkonzept, das die Entstehung eines eigenständigen System- und Komponentenwerks mit eigener Produktverantwortung zum Ziel hatte. Es geht bei den Verhandlungen mit der Unternehmensseite also nicht nur um einen einfachen „Head-Count“, sondern um die Entwicklung eines tragfähigen Konzeptes, das betriebswirtschaftlichen Erfolg und die ökonomische Eigenständigkeit sichert. Erreicht wurde ein Managementbuy-out, wodurch der Standort - wenn auch mit Stellenabbau und entsprechendem Sozialplan - erhalten bleibt.

Verhandlungsorientierte Strategien kommen nicht nur bei ökonomisch günstiger Ausgangslage zum Tragen. Das zeigt das Beispiel des Automobilzulieferers Thyssen Krupp Drauz-Nothelfer. Der von der Schließung bedrohte Ravensburger Standort wies - trotz roter Zahlen des Betriebs eine hohe Produktivität auf. Auch hier drängten IG Metall und Betriebsrat in Verhandlungen darauf, ein tragfähiges ökonomisches Zukunftskonzept zu entwickeln, das den Betrieb für potenzielle Investoren attraktiv machen sollte. Im Februar 2008 wurde das Werk an die ortsansässige Firma Engineering Bausch \& Ziege (EBZ) verkauft. Die Arbeitsplätze bleiben (vorerst) erhalten.

Allerdings: Die verhandlungsorientierte Strategie kann von zwei Seiten unter Druck geraten:

(1) innerhalb der Belegschaft, wenn die Verhandlungen mit der Unternehmensseite $\mathrm{zu}$ stark kompromissorientiert erscheinen oder Teile der Belegschaft weniger auf das Überleben des Betriebs, sondern auf möglichst hohe individuelle Abfindungen setzen;

(2) innerhalb der Interessenvertretung, wenn es zu Widersprüchen zwischen einer gewerkschaftlichen strukturökonomischen
Strategie kommt, die nicht nur den Einzelbetrieb, sondern die regionale Industriestruktur im Blick hat, und einer stärker betriebsökonomischen Ausrichtung der betrieblichen Interessenvertretung, die auf den unbedingten Werkserhalt fokussiert ist.

\subsection{STREIKS FÜR SOZIALTARIFVERTRÄGE}

Obwohl der Erhalt der Arbeitsplätze absolute Priorität für die von Betriebsschließungen bedrohten Belegschaften hat, gibt es vielfach im Laufe der Auseinandersetzung eine Veränderung der Ziele. In gut vier Fünftel der von uns dokumentierten Fälle mündet die Auseinandersetzungen in Vereinbarungen über Abfindungen und die Einrichtung von Beschäftigungs- und Qualifizierungsgesellschaften (BQG). Ein besonders wirksames Instrument, hier gute Konditionen zu erkämpfen, sind Streiks für Sozialtarifverträge. Solche Streiks wurden beispielsweise bei Heidelberger Druck in Kiel, Otis in Stadthagen, Infineon in München, AEG in Nürnberg geführt - Arbeitskämpfe, die schließlich nach BAG-Urteil für rechtens erklärt worden sind und damit die Handlungsinstrumente der Belegschaft sowie der betrieblichen und gewerkschaftlichen Interessenvertretung gegenüber Sozialplanverhandlungen erweitern. Einerseits handelt es sich um ein defensives Instrument: Streikzulässig ist nicht die Verhinderung einer Betriebsschließung, sondern ausschließlich die Milderung der Folgen. Andererseits ermöglicht das Instrument Sozialtarifvertrag eine offensive Arbeitskampfstrategie - anders als Sozialplanverhandlungen mit Einigungsstelle. Erzwingungsstreiks werden möglich, bei denen die Gewerkschaft aktiver Akteur einer betrieblichen Tarifbewegung ist. Beim Sozialtarifvertrag „kommen die allgemeinen Vorteile einer betrieblichen Tarifbewegung zum Zuge: die Beschäftigten können sich viel besser und viel direkter als nach BetrVG in die Auseinandersetzung einbringen. Die Tarifbewegung und das erzielte Ergebnis werden damit zu ,ihrem Ding؛ Man kann mehr Druck machen; wenn nötig, kann die Gewerkschaft legal zu Warnstreiks aufrufen, eine Urabstimmung durchführen, usw." (IG Metall Bezirk Küste o.J.). Allerdings: Ein einheitlicher Diskussionsstand zum Thema „Sozialtarifvertrag" ist in der IG Metall gegenwärtig nicht erkennbar.
Der Perspektivenwechsel von der Verhinderung der Stilllegung zur Verhandlung der Stilllegungskonditionen geht häufig nicht konfliktlos über die Bühne. Das zeigte die Auseinandersetzung bei Bosch-Siemens-Hausgeräte (BSH) in Berlin Spandau: Weil dort der Abbau von knapp 200 Arbeitsplätzen vereinbart wurde, stimmten in der zweiten Urabstimmung nur 35,6\% der organisierten Belegschaft für die Beendigung des Arbeitskampfes. Bei Otis in Stadthagen kritisierten Teile der Belegschaft, dass die IG Metall das Ziel aufgab, den Standort zu sichern. Bei der AEG in Nürnberg wurde das Spannungsverhältnis als offener Strategieprozess thematisiert: als „Doppelstrategie“, die zwei Ziele ansteuerte: Arbeitsplatz- und Standortsicherung wie auch den Abschluss eines Sozialtarifvertrages (Wechsler 2007).

Erkennbare Mobilisierungsprobleme entstehen gleichwohl nicht. Weder Ohnmachtsgefühle noch Fatalismus sind auszumachen, auch kein Nachlassen des Engagements im Kampf für Abfindungen und Beschäftigung in einer Transfergesellschaft.

Beschäftigungs- oder Qualifizierungsgesellschaften haben sich durchaus als ein effektives arbeitsmarktpolitisches Instrument erwiesen. Sie erreichen nach einem Jahr Vermittlungsquoten von etwa $65 \%$ der Beschäftigten. Allerdings sind die BQGs heute - anders als in den 1980er Jahren - nicht in einen politisch gestalteten Strukturwandel eingebettet. Es fehlt eine breitere gesellschaftliche Debatte über eine Wirtschaftssteuerung, die neue Beschäftigungsfelder schafft und Standortkonkurrenzen überwindet.

\subsection{BASISORIENTIERTER PROTEST}

Wenn sich basisorientierte Proteststrategien herausbilden, ist dies nicht zuletzt besonders prekären Ausgangslagen geschuldet: schlechte Arbeitsmarktbedingungen der Beschäftigten und eine geringe ökonomische Verhandlungsmasse in den Betrieben. Der Verhandlungsspielraum wäre folglich eng.

Auf die Marktbereinigungsstrategie der US-Beteiligungsgesellschaft Lone Star im Sommer 2007 antworteten die Beschäftigten von Bike Systems mit einer dreimonatigen Betriebsversammlung (Sicherung des Zugangs zum Betrieb) und einer intensiven Öffentlichkeitsarbeit. Mit der Herstellung des feuerroten „Strike Bike“ nahmen sie selbstorganisiert für eine Woche die Pro- 
duktion wieder auf - nicht nur als symbolischer Akt mit großer öffentlicher und Medienresonanz, sondern zugleich als Demonstration von Produzentenwissen und Produzentenstolz gegen rücksichtslose Kapitalmacht. Bei dieser Aktion ist der Übergang zu einer förmlichen Betriebsbesetzung und zur Aneignung der Produktion fließend. Mit der Eröffnung des Insolvenzverfahrens verließen die Beschäftigten das Werk; 122 Beschäftigte wechselten für acht Monate in eine Transfergesellschaft.

Auch bei Panasonic in Esslingen gab es eine problematische ökonomische Ausgangssituation, in der die Mehrheit der Belegschaft eine Doppelstrategie wie bei AEG explizit ablehnte. Die Proteste radikalisierten sich mit einer 130-tägigen Mahnwache auf dem Werksgelände und schließlich einem Hungerstreik. Es kam zu einer Entfremdung zwischen größeren Teilen der Belegschaft und der IG Metall, die auf die Durchsetzung einer Transfergesellschaft orientierte.

Die Fälle des „basisorientierten Radikalprotestes" ereignen sich nach unserer Recherche dort, wo Unternehmen jegliches Interesse an einer Fortsetzung der Produktion - auch bei erheblicher Kostenreduzierung - oder an einen Verkauf des Werks verloren haben. Die Auseinandersetzungen folgen nicht einem vorab definierten strategischen Plan (wie er im Fall der Streikkonzepte für Sozialtarifverträge notwendig ist), sondern sie entwickeln sich im Kontext dezentraler Willensbildungsprozesse. Träger des Protestes sind in erster Linie lokale Akteure, aus der Belegschaft, unterstützt von selbstorganisierten Solidaritätsgruppen. Was bleibt (und zugleich der Grund dafür ist, dass die Kämpfe von den beteiligten Akteuren insgesamt als Erfolg gedeutet werden) ist die Erfahrung, sich nicht einfach den Unternehmensentscheidungen gebeugt zu haben.

\subsection{INTERNATIONALE VERNETZUNG}

Im global operierenden Finanzmarktkapitalismus lässt sich Widerstand häufig nur im Rahmen einer standortübergreifenden Vernetzungsstrategie entwickeln. Wie schwierig sie zu realisieren ist, zeigte sich jüngst erst bei Nokia.

General Motors Europe dagegen scheint uns ein innovativer Fall zu sein, zumal er sich in einem Kernfeld transnationaler Unternehmensrestrukturierungen abspielt, wo Standortperspektiven im Rahmen kon- tinuierlicher Benchmarkverfahren generiert bzw. zerschlagen werden. Gegen systematische Standortkonkurrenz im Sinne wertorientierter Unternehmenssteuerung wurden bei Opel, Saab und Vauxhall Informations-, Koordinations- und Verhandlungsverfahren im Rahmen des European Employee Forum (EEF) entwickelt. Ein entscheidender Schritt in der Geschichte des EEF war der Abschluss der ersten europäischen Rahmenvereinbarung mit General Motors Europe im Jahr 2000 anlässlich geplanter Ausgliederungen im Bereich Motoren, Getriebe und Einkauf in ein Joint Venture mit FIAT. Hier konnte sich das EEF erstmals als Verhandlungspartner - und nicht mehr lediglich als Informationsgremium - etablieren. Die ersten großen europaweiten Proteste fanden im Jahr 2001 anlässlich der Schließung des Vectra-Werks von Vauxhall in Luton (GB) statt; betriebsbedingte Kündigungen konnten verhindert und der Produktionsstandort Luton erhalten werden. Die Auseinandersetzung im Jahr 2004, bei der insbesondere der Standort Bochum unter Beschuss stand, mündete in einem europaweiten Aktionstag: rund 50.000 Menschen in zehn Ländern protestierten gegen den geplanten Arbeitsplatzabbau. Im Dezember 2004 einigte sich das EEF mit der Europaleitung des Konzerns auf ein Rahmenabkommen, in dem das Unternehmen den Verzicht auf betriebsbedingte Kündigungen und Werksschließungen wie auch eine Zukunftsperspektive für die Marken Opel, Vauxhall und Saab sowie die Einhaltung der bestehenden Tarifverträge zusicherte.

Mit der internationalen Koordination durch das EEF werden vor allem zwei Zielsetzungen verfolgt: Zum einen sollen gemeinsame Positionen erarbeitet werden, um eine gegenseitige „Unterbietungsspira$l^{\prime c} \mathrm{zu}$ verhindern (wie etwa die Vereinbarung des nationalen Flächentarifniveaus als unterste Konzessionsgrenze); zum anderen geht es um die Sammlung und den Austausch von Informationen zu Unternehmensstrategien, was eine möglichst frühzeitige Einflussnahme des EEF ermöglichen soll.

Auch internationale Vernetzungsstrategien bauen auf den Druck durch die - hier internationale - Mobilisierung der Belegschaft. Keine der großen Restrukturierungswellen ging ohne massive Protestaktionen und (Warn-)Streiks an dem einen oder anderen Standort über die Bühne auch wenn dabei aus der Position der De- fensive heraus gekämpft werden muss: "Sharing the pain“ (Haipeter 2006, S. 621; Herber/Schäfer-Klug 2002). Die Verantwortungs- und Entscheidungsverlagerung vom lokalen Betriebsrat auf den Euro-Betriebsrat (EBR) ist der Versuch, den zentralisierten Unternehmensentscheidungen und dem Standortwettbewerb eine entsprechende Organisation auf Seiten der Arbeitnehmer entgegenzusetzen.

\section{Eine "neue Qualität" der Interessensauseinander- setzung}

\subsection{VOM WIDERSTAND ...}

In einer ersten Interpretation der von uns dokumentierten Fälle springt zunächst die neue unternehmensstrategische Qualität von Betriebsschließungen ins Auge: Betriebsschließungen - ehemals Resultate von Krisen und Strukturwandel - sind im globalen Finanzmarktkapitalismus zu einem „normalen“ Instrument unternehmenspolitischer Restrukturierung geworden.

Sehr viel schwerer fällt eine Einschätzung der Kämpfe gegen Betriebsschließungen und Standortverlagerungen. Sie sind zumeist der Höhepunkt eines schon lange andauernden Abwehrkampfes, in dem angesichts eines unternehmerischen Schließungs- und Verlagerungsszenarios viele der früheren sozialen Errungenschaften für ausgehandelte Beschäftigungssicherung geopfert wurden. Ihre teilweise Radikalität ist auch Ausdruck der Ohnmacht und der Empörung über die einseitige Aufkündigung der eingeübten sozialpartnerschaftlichen, konsensorientierten Interessensvertretungspolitik. Eine Politik der Zugeständnisse und Konzessionen ist sichtbar an einem Ende angelangt. Es wird eine Grenze überschritten, die das eingespielte Verhältnis der Akteure der Interessenauseinandersetzung infrage stellt. Meistens gemeinsam mit Betriebsräten und Gewerkschaften, manchmal auch in kritischer Distanz zu ihnen, entwickeln sich Widerstandsformen von unten, die durchaus als eine „neue Kultur der Beteiligung“ und angesichts der festgefahrenen Stellvertreterpolitik der letzten Jahrzehnte als „eine kleine Revolution“ (Candeias/Röttger 2007) bezeichnet werden 
können. ${ }^{2}$ Auch wenn wir bislang wenig über die individuellen und kollektiven Orientierungen und Bewusstseinsformen bei diesen Kämpfen wissen, belegen die Kämpfe an sich und die dokumentierten Formen des Widerstands eine neue Qualität der Interessenauseinandersetzung. Widerstand ist keinesfalls zwecklos, wie Beck meint. Dies gilt auch jenseits der tatsächlich erreichten materiellen Ergebnisse.

Auch wenn nur in wenigen Fällen der Standort erhalten oder zumindest für Teile der Belegschaft die Beschäftigung gesichert werden konnte, so zeigt sich doch, dass auch zu Zeiten eines scheinbar übermächtigen Finanzmarktkapitalismus beides nicht völlig unmöglich ist. Denn das ist der Unterschied $\mathrm{zu}$ früheren Formen der Betriebsschließungen: Nicht nur Belegschaften sind erpressbar, auch gesunde, aber nach kurzfristigen Renditeerwartungen gesteuerte Unternehmen lassen sich durch massiven Widerstand unter Druck setzen. Und sie haben trotz neoliberaler Hegemonie Legitimationsprobleme, wie die erreichte öffentliche Aufmerksamkeit und die breite Unterstützung der Kämpfe im lokalen Umfeld zeigen.

In den meisten Fällen bestand der Erfolg in der sozialen Abfederung des Beschäftigungsabbaus, einschließlich der Maßnahmen, um die Arbeitsmarktchancen der Beschäftigten durch ihren Übergang in Transfergesellschaften zu verbessern. Um deren Ausstattung wird intensiv gekämpft. Das Instrument des Sozialtarifvertrags eröffnet eine neue Kampfform, die vorhandene Widerstandsbereitschaft wirksam zu mobilisieren. Die hiermit erreichbaren Ergebnisse bleiben dennoch Ausdruck einer defensiven Perspektive. Die sozialen Konsequenzen von Betriebsschließungen werden abgemildert, Übergänge in neue Beschäftigung erleichtert. Der Preis, den die Unternehmen zu zahlen haben, wird hochgetrieben, in den meisten Fällen jedoch nicht so hoch, dass sie auf Schließung bzw. Verlagerung verzichten.

\section{2 ...ZUR ANEIGNUNG}

Der defensive Charakter der Kämpfe wird dann durchbrochen, wenn versucht wird, Einfluss auf die unternehmerischen Entscheidungen zu nehmen, auf die Investitions- und Produktpolitik, die - zum Teil verdeckt durch vordergründige Kostenargumente - letztlich für die Schließungsab- sichten verantwortlich sind. Hinter diesen Versuchen steckt zunächst keine neue betriebs- oder gewerkschaftspolitische Strategie. Sie folgen eher der inneren Logik der unternehmerischen Strategien, die eine weitergehende Einflussnahme ,erzwingen“. Es ist die schlichte Einsicht, dass den sozialen Konsequenzen von Betriebsschließungen und -verlagerungen nur dann wirkungsvoll begegnet werden kann, wenn möglichst frühzeitig auf den Kern der Unternehmenspolitik, die Investitions- und Produktentscheidungen, Einfluss genommen wird. Bei Betriebsschließungen werden dieser Zusammenhang und damit auch die Grenzen bestehender Mitbestimmungsrechte in unmittelbarer Weise deutlich. Das zeigt sich besonders dort, wo Betriebsräte und Gewerkschaften mit alternativen Unternehmens- und zum Teil auch Branchenkonzepten in eine Auseinandersetzung gehen, bei der es nicht mehr nur um das $\mathrm{Ob}$ der Produktion geht, sondern auch um das Was, das Wie und das Wieviel. Sie können auf der Basis von Alternativgutachten zeigen, dass es durchaus eine Zukunft für den Standort oder die Region gibt, und beteiligen sich auch an der Suche nach neuen Investoren. Diese „Aneignung des Unternehmerischen" geht mit ihrer offensiven Perspektive über traditionelles Co-Management hinaus, vor allem dann, wenn sie nicht auf der Ebene einer technokratischen Stellvertreterpolitik verbleibt. Denn die Stärke der Verhandlungsposition hängt davon ab, in welchem Umfang die Belegschaft und Öffentlichkeit Druck auszuüben vermögen.

Die Spannung zwischen Basismobilisierung und ergebnisorientierter Verhandlung führt zu einer Vielzahl von Konflikten, vor allem dann, wenn die Gewerkschaften die Mobilisierung der Belegschaft in einer eher instrumentellen Perspektive sehen. In der Tradition bisheriger Interessenvertretungspolitik stehen immer noch die „ökonomische Vernunft" und ein am materiellen Erfolg orientierter Pragmatismus im Vordergrund. Die Protestbedürfnisse der Beschäftigten und zu erreichende Mobilisierungs- und Politisierungserfolge werden dementsprechend geringer eingeschätzt.

Das zeigt sich auch im Umgang der Gewerkschaften mit basisorientierten Konfliktverläufen, in denen es - bei meist ungünstigeren ökonomischen Voraussetzungen - neben radikaleren Protestformen auch zu einer „unmittelbaren Aneignung des Unternehmerischen" kommt: Auch hier geht es um alternative Produkt- und Produktionskonzepte, die symbolisch mit der Besetzung und der selbst organisierten Übernahme der Produktion unterstrichen werden. Die materiellen Erfolge dieser Kämpfe waren gering, die Politisierungseffekte einer bislang unerfahrenen und kaum organisierten Belegschaft und die Aufmerksamkeit der Öffentlichkeit waren jedoch vergleichsweise groß. Die Politisierung ging über betriebspolitische Ziele hinaus und richtete sich z. B. auch gegen Sozialabbau und andere neoliberale Politikformen. Das Verhältnis zwischen den Beschäftigten und den Gewerkschaften war hierbei eher distanziert bzw. von Konflikten geprägt.

Solche Konflikte sind auch ein Indikator dafür, dass bei den Kämpfen gegen Betriebsschließungen Bewegung in das traditionelle Gefüge der Beziehungen zwischen Belegschaften und ihrer betrieblichen und gewerkschaftlichen Interessenvertretung gekommen ist. Auch das verweist auf eine neue Qualität in der Interessenauseinandersetzung: Neue Formen des Widerstands, der Mobilisierung und Politisierung von Belegschaftsinteressen sind die eine Seite, eine neue Form der Aneignung des Unternehmerischen, eine Ausweitung von Mitbestimmungsforderungen, Einflussnahme auf Investitions- und Produktentscheidungen die andere Seite. Dies muss nicht auf der Ebene des Einzelbetriebs verbleiben. Eine Aneignung des Unternehmerischen im Rahmen alternativer Unternehmenskonzepte kann sich mit den sozialen Auseinandersetzungen um eine ökonomische Regulation auf sektoraler und nationaler Ebene verbinden.

Politische Sprengkraft und eine wirkungsvolle Stärkung von Arbeitnehmerpositionen entstehen dann, wenn beide Seiten zusammen kommen: wenn Widerstand und Mobilisierung eine inhaltliche Perspektive erhalten und wenn alternative Produkt- und Produktionskonzepte nicht nur das Argument der „ökonomischen Vernunft" für sich, sondern auch eine kampfbereite Belegschaft und eine aufmerksame Öffentlichkeit hinter sich haben.

\footnotetext{
2 Diese etwas vollmundige Einschätzung greift aus unserer Sicht allerdings zu kurz, wenn die "revolutionäre Qualität" allein auf neue Beteiligungsformen bezogen wird. Ebenso wichtig ist die inhaltliche Perspektive, in der über den Widerstand hinaus neue Aneignungsformen entwickelt werden.
} 
Die von uns betrachteten Fälle dokumentieren, dass es gehen könnte. Dazu ist vieles erforderlich: auf der Akteursebene vor allem eine widerständige Belegschaft, eine belastbare Zusammenarbeit von Beschäftigten, Betriebsrat und Gewerkschaft, eine aufgeschlossene Öffentlichkeit sowie zivilgesellschaftliche und politische Bündnispartner; auf der konzeptionellen und strategischen Ebene das, was wir als Aneig- nungsperspektive im Hinblick auf das Was, Wie und Wofür der Produktion umrissen haben. Von beidem gehen Lernprozesse aus. Dazu könnte - und sollte aus unserer Sicht - gehören, in den Gewerkschaften eine Öffentlichkeit für die zumeist lokal begrenzten, über die Region und die Verwaltungsstellen hinaus oft nicht bekannten Kämpfe gegen Betriebsstilllegungen herzustellen. Wenn unsere These eines neuen
Typs von Betriebsstilllegungen und Standortverlagerungen im Finanzmarktkapitalismus richtig ist, sollte dies Anlass für breiter angelegte gewerkschaftliche Aufarbeitung und Strategiedebatten sein. So könnten die Kampferfahrungen von Beschäftigten und Interessenvertretungen als Ermutigung dienen und dazu beizutragen, ihre Erfolgschancen bei Standortkonflikten künftig zu verbessern.

\section{LITERATUR}

AEG (2006): Der AEG-Streik in Nürnberg. Der erfolgreiche Kampf für einen Sozialtarifvertrag, (Hrsg.): IG Metall Bezirksleitung Bayern, IG Metall VWSt Nürnberg, IG Metall VWSt. Köln, München-Nürnberg-Köln

Ahlers, E./Öz, F./Ziegler, A. (2007): Standortverlagerung in Deutschland - einige empirische und politische Befunde, edition Hans-Böckler-Stiftung 194, Düsseldorf

Beck, U. (2007): Und jetzt, Herr Beck?, in: Geiselberger, H. (Hrsg.): Und jetzt? Politik, Protest und Propaganda, Frankfurt a. M., S. 231-248

Bierbaum, H. (2006): AEG: Auf dem Altar des "shareholder value", in: AEG 2006, S. 92-95

Candeias, M./Röttger, B. (2007): Sozialtarifverträge und lokale Arbeiterbewegungen, in: Geiselberger, H. (Hrsg.): Und jetzt?, Politik, Protest und Propaganda, Frankfurt a. M., S. 88-96

CNH (2006): Berlin ist O\& K!. Der Streik bei der CNH-Baumaschinen $\mathrm{GmbH}$ in Berlin Spandau, (Hrsg.): IG Metall Bezirk Berlin-BrandenburgSachsen, Berlin

Haipeter, T. (2006): Der Europäische Betriebsrat bei General Motors Auf dem Weg zur europäischen Mitbestimmung?, in: WSI-Mitteilungen 11, S. 617-623

Harvey, D. (2005): Der neue Imperialismus, Hamburg
Herber, A./Schäfer-Klug, W. (2002): Wie ein Eurobetriebsrat zum Vertragspartner wird, in: Mitbestimmung 9, S. 50-54

Heseler, H. (1990): Die Arbeitsmarktfolgen von Betriebsschließungen. Erfahrungen im internationalen Vergleich, in: MittAB 3, S. 410-421

IG Metall Bezirk Küste (o. J.): Positionspapier, o. O.

Infineon (2006): Streik für einen Sozialtarifvertrag. Infineon München Perlach, (Hrsg.): IG Metall Bezirk Bayern, München

Iwer, F. (2006): Weiße Ware - Besonderheiten der Branche, in: AEG

2006, S. 84-87

Lutz, B. (1984): Der kurze Traum immerwährender Prosperität - Eine Neuinterpretation der industriell-kapitalistischen Entwicklung in Europa des 20. Jahrhunderts, Frankfurt/New York

Marx, K. (1972): Das Kapital, Band 1, MEW 23, Berlin

Otis (2005): Streik für einen Ergänzungs-Tarifvertrag. Eine neue Kampfform gegen den Profitwahn der Globalisierung, (Hrsg.): IG Metall, Stadthagen

Voskamp, U. (2005): Grenzen der Modularität - Chancen für Hochlohnstandorte in globalen Produktions- und Innovationsnetzwerken, in: SOFIMitteilungen 33, Göttingen, S. 115-129

Wechsler, J. (2007): Der Streik um den Sozialtarifvertrag bei AEG, in: Bispinck, R. (Hrsg.): Wohin treibt das Tarifsystem?, Hamburg 